\title{
Hißmann und der Materialismus
}

Michael Hißmann gehört zu der vergleichsweise kleinen Gruppe deutscher Philosophen des 18. Jahrhunderts, die sich in ihren Publikationen ausdrücklich zum Materialismus bekennen. Ganz abgesehen davon, dass es unterschiedliche Versionen der materialistischen Position gibt, hat das, was jeweils `Materialismus` oder heute >Physikalismus` heißt, mehrere Aspekte und lässt sich nicht auf eine These reduzieren. ${ }^{1}$ In diesem Beitrag geht es um ein wesentliches Lehrstück, das auch für Hißmann zentral ist: die Lehre von der Materialität der menschlichen Seele oder des menschlichen Geistes. Es gilt, erstens, Hißmanns Beitrag im Kontext des 18. Jahrhunderts zu erläutern, und, zweitens, seine Argumente, mit denen er seine Position zu begründen sucht, $\mathrm{zu}$ analysieren und kritisch zu würdigen.

\section{Hißmann im Kontext}

Im 18. Jahrhundert gab es bekanntlich mehrere Debatten zur materialistischen Auffassung vom menschlichen Geist oder von der Seele. Diese Auffassung wurde oft unter dem Titel der >denkenden Materier abgehandelt und erhielt ab etwa der Jahrhundertmitte zumindest stillschweigende Unterstützung durch die verstärkt auftretenden Versuche, die Wirkungen des menschlichen Geistes allein auf Grundlage einer (freilich sehr spekulativ verfahrenden) Physiologie zu erklären, und durch die Entwicklung eines neuen Materiebegriffs, demgemäß die Materie nicht länger als wesentlich passiv und durch Trägheit zu charakterisieren ist, sondern als etwas, das selbst aktive Kräfte enthält. ${ }^{2}$ Besonders einflussreich waren in diesem Zusammenhang die Arbeiten von Denkern wie Charles Bonnet und David Hartley. Zwar distanzieren sich beide ausdrücklich vom Materialismus, aber ihre Erklärungsweisen mentaler Phänomene tendieren trotz

1 Vgl. hierzu beispielsweise Kurt Bayertz: Was ist moderner Materialismus? In: Kurt Bayertz, Myriam Gerhard und Walter Jaeschke (Hg.): Weltanschaunng, Philosophie und Naturwissenschaft im 19. Jahrbundert. Band 1: Der Materialismus-Streit. Hamburg 2007, S. 50-70.

2 Vgl. John W. Yolton: Thinking Matter. Materialism in Eighteenth-Century Britain. Minneapolis 1983, pp. 90-106, 153-189. Yolton weist zudem darauf hin, dass der Einfluss von »Locke's suggestion«, wonach der Begriff einer denkenden Materie widerspruchsfrei gedacht werden könne, für die Entwicklung des Materialismus im 18. Jahrhundert von zentraler Bedeutung gewesen sei (pp. 14-28). 
aller Beschwörungen einer immateriellen Seele deutlich zur materialistischen Position. ${ }^{3}$ Denn Hartleys Darstellung der Entstehung von Empfindungen, Ideen und Bewegungen bedient sich ausschließlich seiner physiologischen Psychologie und macht dadurch die Annahme einer immateriellen Seele de facto überflüssig. ${ }^{4}$ Dies ist genau jener Aspekt der Hartleyschen Lehre, an den seine materialistisch denkenden Nachfolger anknüpfen. Joseph Priestley, zweifellos einer der wichtigsten materialistischen Denker des 18. Jahrhunderts, beruft sich denn auch ausdrücklich auf Hartleys Theorie, der er eine begründende Funktion für seine eigene materialistische Theorie des Geistes zuspricht. ${ }^{5}$ Dies ergibt sich bereits aus seiner gekürzten Fassung von Hartleys Observations on Man, die er 1775 in London unter dem Titel Hartley's Theory of the Human Mind veröffentlichte. Priestley fügt dieser Edition drei einführende Abhandlungen hinzu, in denen es vor allem um Hartleys Vibrationstheorie, die Assoziationslehre und das Problem der komplexen und abstrakten Ideen geht und in denen er die materialistischen Tendenzen bei Hartley betont. In den Diskussionen der Folgezeit wird Hartleys Assoziationslehre meist mit Priestleys Materialismus in Verbindung gebracht, was sicher nicht in Hartleys Sinn gewesen wäre.

Im deutschsprachigen Raum rezipiert Albrecht von Haller schon früh Hartleys Theorie, ${ }^{6}$ doch ihre Breitenwirkung setzte erst in den 1770er Jahren ein. In den Jahren 1772/3 erschien bereits eine deutsche Übersetzung von Hartleys Observations mit kritischen Anmerkungen von Hermann Andreas Pistorius, die aber nur den zweiten, weitgehend theologischen Teil enthält und lediglich eine kurze Zusammenfassung des ersten, theoretischen Teils bietet. Die Tatsache, dass Hartleys Theorie dennoch in der deutschen empirischen Psychologie der 1770er Jahre gegenwärtig war, ist einer deutschen Übersetzung der Essays zu verdanken, die Priestley seiner Hartley-Edition vorangestellt hatte. Diese Übersetzung wurde von Michael Hißmann im ersten Band als erste Abhandlung seines Magazins für die Philosophie und ihre Geschichte publiziert. ${ }^{7}$ Damit lag, dank Hißmann, dem deutschen Publikum eine konzise, materialistisch interpretierte Version von Hartleys Theorie des Geistes vor, die auch eingehend rezipiert wurde.

Des Weiteren orientiert sich Hißmanns eigene materialistische Position stark an Priestley, wie wir sehen werden. Als äußeres Indiz für Hißmanns Priestley-tum lässt sich angeben, dass er den drei Abhandlungen Priestleys in seiner Übersetzung denselben Titel gibt wie seinem eige-

3 David Hartley: Observations on Man, His Frame, His Duty, and His Expectations (1749), zitiert nach der 6. Auflage. London 1834; Charles Bonnet: Essai analytique sur les facultés de l'ame. Kopenhagen 1760.

4 Vgl. beispielsweise die Darstellung von Hartley in Udo Thiel: Erkenntnistheorie und Psychologie im Gefolge Lockes. In Helmuth Holzhey (Hg.): Grundriss der Geschichte der Philosophie. 18. Jahrbundert. England Nordamerika. Basel 2004, S. 403-410.

5 Priestleys wichtigstes Werk zum Materialismus ist Disquisitions relating to Matter and Spirit, London 1777. Er beruft sich explizit auf Hartley in seiner Kritik an Thomas Reid und der Philosophie des Common Sense. Vgl. Priestley: An Examination of Dr. Reid's Inquiry into the Human Mind on the Principles of Common Sense, Dr. Beattie's Essay on the Nature and Immutability of Truth, and Dr. Oswald's Appeal to Common Sense in Behalf of Religion. London 1774, pp. xi-xiii.

6 Vgl. Albrecht von Hallers Rezension von Hartleys Observations on Man. In: Göttingische Gelehrte Anzeigen (1750), S. 212-213, 232-233.

7 Michael Hißmann (Hg.): Magaz̧in für die Philosopbie und ibre Geschichte, Bd. 1, Göttingen und Lemgo 1778, S. 7-60. Im Original erschienen diese Essays unter dem Titel Introductory Essays. Vgl. Joseph Priestley (ed.): Hartley's Theory of the Human Mind. London 1775, pp. ix-li. 
nen Hauptwerk: Psychologische Versuche. ${ }^{8}$ Hißmann fordert im Sinne seines Göttinger Kollegen Christoph Meiners eine Revision der Philosophie, so der Titel von Meiners’ Werk von 1772. Gemäß dem Projekt einer solchen Revision hat sich die Philosophie auf eine empirische Psychologie zu gründen, und diese ist wiederum auf eine materialistische, das heißt rein physiologische Grundlage zu stellen.

\section{Hißmann und Materialismen - Methodisch, Metaphysisch, Identi- tätstheoretisch, Eliminativ?}

Der Materialismus ist hier sowohl als eine metaphysische als auch als eine methodologische Position zu verstehen. ${ }^{9}$ Denn dieser besagt erstens, dass es keine immateriellen Entitäten gibt; alles, was existiert, ist materiell. Hieraus ergibt sich zweitens, dass alles, was existiert, einer bestimmten Untersuchungsmethode unterworfen werden kann, nämlich der empirischen Methode von Beobachtung und Experiment, das heißt der Naturwissenschaften, und im Falle der menschlichen Seele oder des Geistes, der physiologischen Psychologie. Ganz ähnlich sieht übrigens die Sachlage im späten 20. und im 21. Jahrhundert aus. Paul Churchland etwa spricht in seinem einflussreichen Werk Matter and Consciousness durchaus von einem ontologischen Problem, andererseits aber auch von semantischen, erkenntnistheoretischen und methodologischen Problemen. ${ }^{10}$ Das heißt, es wäre falsch, einen Gegensatz zwischen dem Materialismus des 18. Jahrhunderts und dem Materialismus oder Physikalismus der Gegenwart dadurch zu konstruieren, dass man dem einen eine reine methodische Ausrichtung zuspricht, dem anderen eine bloß substanzmetaphysische. Beide Aspekte sind relevant, sowohl heute als auch im 18. Jahrhundert. So schreibt Hißmann in den Psychologischen Versuchen: »Ist die menschliche Seele Materie [metaphysische These]: so müssen wir nothwendig eine neue Psychologie haben, die nur der physiologische und anatomische Psycholog schreiben kann « [methodologische These]. ${ }^{11}$ Immer wieder betont Hißmann methodische Aspekte, insbesondere die Bedeutung von »Beobachtungen und Erfahrungen«. Zu den Vorgängern auf diesem methodischen Weg zählt er außer Priestley und Hartley auch Condillac, Helvétius, Bonnet, Search (i. e. Abraham Tucker), Robinet und Lossius. Dagegen sei die »Monadologie« ein »metaphysischer Traum«, und, so frohlockt Hißmann, »demonstrativische Pedanterie« sei mehr und mehr »aus dem aufgeklärten Europa

8 Michael Hißmann: Psychologische Versuche, ein Beytrag zur esoterischen Logik. Frankfurt und Leipzig 1777. Eine zweite Auflage erschien postum 1788.

9 Auf die Unterscheidung zwischen methodischem und metaphysischem Materialismus macht Michael Pauen aufmerksam. Michael Pauen: Vom Streit über die Seelenfrage bis zur Erklärungslücke. Wissenschaftlicher Materialismus und die Philosophie der Naturforscher im Vergleich mit dem Physikalismus der Gegenwart. In: Kurt Bayertz, Myriam Gerhard und Walter Jaeschke (Hg.): Weltanschaunng, Philosophie und Naturwissenschaft im 19. Jahrhundert. Band 1: Der Materialismus-Streit. Hamburg 2007, S. 102-125. Vgl. auch Falk Wunderlich, der auf Pauen verweist: Johann Georg Sulzers Widerlegung des Materialismus und die Materietheorien der Zeit. In: Frank Grunert und Gideon Stiening (Hg.), Johann Georg Sulzer (1720-1779). Aufklärung zwischen Wolff und Hume, Berlin 2011, S. 37-55, hier S. 39.

10 Paul M. Churchland: Matter and Consciousness. A Contemporary Introduction to the Philosophy of Mind, Revised Edition, Cambridge, Mass. 1988. 
verbannet worden «. ${ }^{12}$ Vielmehr gelte: »Nun samlet man Fakta, man giebt auf ihre Folgen Acht [...] man hat Fakta, man hat Erklärungen «. ${ }^{13}$

Die neue Methode verlangt nach Hißmann auch die Schaffung eines neuen Typs von Wissenschaftler, der Naturwissenschaft mit Philosophie verbindet:

Die Sammlung dieser Thatsachen; behutsame Folgerungen aus denselben würden alles bisherige psychologische Gewäsche an Vorzügen und Brauchbarkeit unendlich überwiegen: denn sie würden den offenbaren, seltenen Vorzug der Gründlichkeit haben. Aber der Philosoph müste Arzt, und der Arzt Philosoph seyn; und folglich eine neue Art von Kreaturen entstehen. ${ }^{14}$

Es müssten mit der Seele auch Experimente gemacht werden, so sagt Hißmann, insbesondere Krankheitszustände müssen ausgenutzt werden, daher auch die Betonung des Arztes. »Physio$\log$ und Anatom müsse man werden, um Psycholog zu seyn. «15 Wenn relevante Krankheitszustände nicht vorliegen, sollte man den Körper durchaus in einen außerordentlichen Zustand versetzen, »um an der Seele Experimente zu machen«. Dies schlagen jedenfalls: »verständigere Psychologen « vor, und sie tun dies laut Hißmann »mit Recht «. ${ }^{16}$

Es stellt sich jedoch die Frage, was für eine Art von materialistischer Theorie des Geistes Hißmann vertritt. Wie eingangs angedeutet unterscheidet man heute zwischen mehreren Versionen des Materialismus oder Physikalismus. Hier seien zwei Beispiele aus dem späten 20. Jahrhundert wenigstens erwähnt, die auch weiterhin diskutiert werden: die Identitätstheorie, die vor allem durch David Armstrong in den 1970er Jahren Furore machte, ${ }^{17}$ und - noch radikaler - der eliminative Materialismus, wonach man Wünsche, Hoffnungen, Gefühle und so weiter nicht mit Gehirnzuständen als identisch bezeichnen sollte, sondern vielmehr gilt, dass es diese Wünsche, Hoffnungen etc. gar nicht gibt. Dass es sie gebe, sei genauso eine Illusion wie es früher der Glaube an Hexen gewesen sei. Es gebe nur die neuronalen Zustände, unser Alltagsverständnis sei nichts als folk psychology, die es zu überwinden gelte. ${ }^{18}$ Für einen von diesen beiden Positionen zu unterscheidenden nicht-reduktiven Materialismus dagegen hängen geistige Phänomene zwar von der Materie ab, sind aber nicht vollständig auf diese reduzierbar und haben damit eine gewisse Eigenständigkeit.

Hißmann kannte diese Unterschiede nicht, und es ist wohl müßig zu versuchen, ihn auf eine der modernen Versionen festlegen zu wollen. Jedenfalls scheint er keinen Eliminativismus zu vertreten, da er an dem Alltagsverständnis von mentalen Zuständen durchaus festhält. Biswei-

11 Hißmann: Psychologische Versuche (s. Anm. 8), S. 11.

12 Ebd., S. 250 (die letzten beiden Zitate).

13 Ebd., S. 251.

14 Ebd., S. $21 \mathrm{f}$.

15 Ebd. S. 250.

16 Ebd.

17 David Armstrong: A Materialist Theory of the Mind. London 1968.

18 Churchland verteidigt den eliminativen Materialismus in Matter and Consciousness (s. Anm. 10, p. 48): »The bald statement of eliminative materialism is that the familiar mental states do not really exist. $\mathrm{Zu}$ argumentieren, dass »introspection reveals directly the existence of pains, beliefs, desires, fears and so forth«, bedeutet nur, dass man »makes the same mistake that an ancient or medieval person would be making if he insisted that he could just see with his own eyes that the heavens form a turning sphere, or that witches exist« (p. 47). Vgl. auch in deutscher Sprache Paul M. Churchland: Die Seelenmaschine. Eine philosophische Reise ins Gehirn. Heidelberg 1997. 
len scheint er das Verhältnis von Mentalem zum Materiellen als ein Abhängigkeitsverhältnis zu verstehen, ohne allerdings die Natur dieser Abhängigkeit zu erläutern. So heißt es beispielsweise einmal, es gelte, dass »die Bezeichnung der Gedanken, sowie die Gedanken selbst [...] von der Disposition des Gehirns abhangen «. ${ }^{19}$ Diese Formulierung deutet darauf hin, dass mentale $\mathrm{Zu}$ stände für ihn nicht als identisch mit Gehirnzuständen anzusehen sind. An anderer Stelle heißt es dagegen: »Man kann [...] den Ausdruck, Seele, und Seelenkräfte immer beybehalten, wenn man unter letztern nichts als Anspannungen der Gehirnorganen, der intellektuellen Fibern versteht, deren verschiedene Modifikationen und Dispositionen verschiedene Begriffe und Ideen sind. $\ll^{20}$ Hier scheint eine Identität von mentalem Zustand und Gehirnzustand behauptet zu werden. Mentale Phänomene sind hiernach einfach als eine bestimmte Art von physischen Phänomenen aufzufassen. Da Hißmann selbst aber nicht zwischen mehreren Materialismen unterscheidet, fragt es sich jedoch, wie wörtlich diese Formulierungen zu nehmen sind. Aus heutiger Sicht mag es jedenfalls als ein Manko angesehen werden, dass Hißmann das Verhältnis von physischer Basis zum Mentalen nicht präzisiert und nicht mehrere Möglichkeiten auf materialistischer Grundlage voneinander unterscheidet, um sich dann für die eine oder andere Version auszusprechen. Aber aus Hißmanns Perspektive sind solche Feinheiten ohnehin nicht wesentlich. Ihm geht es vor allem darum, die traditionelle Lehre von einer immateriellen Seelensubstanz zurückzuweisen und methodisch für eine empirische und physiologische Psychologie als angemessene Herangehensweise beim Studium unserer Seele zu argumentieren.

Darüber hinaus gesteht Hißmann zu, dass seine materialistische Rhetorik durchaus über das hinausgeht, was er mit seinen Argumenten beweisen kann. Es geht ihm letztlich auch gar nicht um einen Beweis, eine Demonstration der Materialität der Seele, sondern nur darum, diese Position als sehr wahrscheinlich auszuweisen. So betont Hißmann: »Wenn einige Stellen in meiner Schrift etwas affirmatif scheinen sollten«, so gilt doch, »dass ich nemlich auf keine Demonstration ausgehe, sondern blos das mehr Wahrscheinliche gegen das Unwahrscheinliche abzuwiegen suche«.21 Auch in dieser Hinsicht besteht eine Ähnlichkeit zu Priestley. Denn obwohl Priestley mit Nachdruck dafür plädiert, dass der Sitz des Denkens das Gehirn sei, sucht er diese These nicht zu beweisen, sondern argumentiert lediglich für ihre Wahrscheinlichkeit. Sein Argument besteht kurz gesagt darin, dass, soweit wir es eben beurteilen könnten, das Denkvermögen und ein bestimmter Zustand des Gehirns immer zusammen vorkommen und einander entsprechen, und dass wir Vermögen und Eigenschaften immer auf Grund solcher Entsprechungsverhältnisses einer Entität (Substanz) zuschreiben. ${ }^{22}$ Ebd., S. 252; die letzten beiden Hervorhebungen von mir.

Ebd., S. 18.

22 »As far as we can judge, the faculty of thinking, and a certain state of the brain, always accompany and correspond to one another; which is the very reason why we believe that any property is inherent in any substance whatsoever.« (Priestley: Disquisitions [s. Anm. 5], p. 27). 


\section{Hißmanns Materialismus und die Religion}

In einer Hinsicht unterscheidet sich Hißmann ganz deutlich vom Materialismus der Gegenwart und auch von anderen Materialisten seiner Zeit. Denn er hält seinen Materialismus für vereinbar mit der Religion und insbesondere mit der Lehre von der Unsterblichkeit der Seele und einem Leben nach dem Tode. Hierin folgt er wiederum Priestley. Priestley hält Materialismus und christlichen Glauben nicht nur für vereinbar, sondern er behauptet sogar, dass das wahre Christentum materialistisch sei. Der Gedanke einer den Körper überlebenden immateriellen Seele sei dem Christentum ursprünglich ganz fremd gewesen und erst später aus der orientalischen und griechischen Philosophie in das christliche Denken eingeführt worden. ${ }^{23}$ Der Gedanke einer Vereinbarkeit von Materialität und Unsterblichkeit der Seele war natürlich nicht neu. In Bezug auf neuzeitliches Denken hätte sich Hißmann beispielsweise auf Locke berufen können, den er oft in anderen Zusammenhängen preist. Denn Locke argumentierte, dass die Hoffnung auf ein Leben nach dem Tode ganz unabhängig von Beweisen für die Immaterialität der Seele sei. ${ }^{24}$ Locke sprach sich zwar nicht für den Materialismus aus, er macht diesen aber mit der Unsterblichkeitslehre kompatibel. Dementsprechend sagt Hißmann, man habe fälschlicherweise gelernt, die Hoffnung auf Unsterblichkeit nur auf »die erdichtete, oder wenigstens zweifelhafte Immaterialität der Seele zu bauen«. ${ }^{25}$ Die Religion, die Hißmann deutlich von der theologischen Dogmatik unterscheiden will, bedürfe des Lehrstücks von der einfachen und unsterblichen Seele gar nicht. Die »Hoffnung auf Unsterblichkeit« werde »nicht erschüttert« durch Zweifel an der Einfachheit der Seele. ${ }^{26}$ Daher kann Hißmann deklarieren: »Ich glaube an die Unsterblichkeit meiner Seele, und ich glaube eben so zuversichtlich an ihre Materialität. «17

In explizitem Anschluss an Hume argumentiert Hißmann allerdings auch gegen die übliche Einstellung, wonach bei Untersuchungen über das Wesen der Seele stets zu fragen ist, ob das Ergebnis mit Unsterblichkeit und Moral übereinstimme. »Nie darf man eine Lehre wegwerfen, die die tägliche Erfahrung lehrt, wenn sie gleich wegen der Vorurteile, die unsern Verstand benebeln, schwer zu verdauen wäre«. ${ }^{28}$ Er beklagt, dass das Problem metaphysischer Untersu-

23 Priestley: Disquisitions (s. Anm. 5), S. 155-156. Vgl. zu Priestely und anderen Versuchen, materialistische Metaphysik mit der Lehre vom Leben nach dem Tode zu vereinbaren Udo Thiel: Religion and Materialist Metaphysics. Some Aspects of the Debate about the Resurrection of the Body in Eighteenth-Century Britain. In: Ruth Savage (Ed.): Pbilosophy and Religion in Enlightenment Britain. New Case Studies. Oxford 2012, pp. 90-111. Priestley's Schüler Thomas Cooper publizierte eine Abhandlung mit dem Titel The Scripture Doctrine of Materialism. Philadelphia 1823.

24 John Locke: An Essay concerning Human Understanding. Ed. by Peter H. Nidditch. Oxford 1975, IV.iii.6 (p. 542): »All the great Ends of Morality and Religion, are well enough secured, without philosophical Proofs of the Soul's Immateriality. A Auch David Hartley spielt die Verbindung von Immaterialität und Unsterblichkeit herunter: »It is most worthy of notice, that the immateriality of the soul has little or no connexion with its immortality." (Hartley: Observations on Man [s. Anm. 3], p. 321).

25 Hißmann: Psychologische Versuche (s. Anm. 8), S. $252 \mathrm{f}$.

26 Ebd., S. 253.

27 Ebd., S. 13.

28 Hißmann: Psychologische Versuche (s. Anm. 8), S. 257f.). Hißmann lobt Humes »Scharfsinn« in diesem Zusammenhang und zitiert anerkennend aus Abschnitt VIII der Enquiry concerning Human Understanding (S. 258). Vgl. David Hume: Enquiries concerning Human Understanding and concerning the Principles of Morals. Ed. by Lewis A. Selby-Bigge a. Peter H. Nidditch. Oxford 1975: »There is no method of reasoning 
chungen in der Vergangenheit gerade darin bestand, dass die Frage nach dem Wesen der Seele mit der Frage nach Leben nach dem Tode verknüpft wurde. Aus dieser Verknüpfung ergab sich, dass die Untersuchungen über die Seele »partheyisch« wurden. »Man muste den Theologen [...] gefallen« ${ }^{29}$ Dank dieser Parteilichkeit habe sich das Lehrstück von der Einfachheit und Immaterialität der Seele überhaupt durchsetzen können. Hißmann sieht sich dagegen nicht mehr gezwungen, den Theologen zu gefallen, und preist den neuen »Geist der Verträglichkeit«, den Geist der »Friedfertigkeit und Duldung«, der »fast im ganzen aufgeklärten Europa« herrsche. ${ }^{30}$

Hißmanns Strategie in Bezug auf die Religion ist demnach zweigleisig: Einerseits argumentiert er, dass die Bewertung der materialistischen Position sich nur auf philosophischer Argumentation zu gründen habe, nicht auf von der Philosophie unabhängige, angebliche Folgen dieser Position für die Religion. Andererseits argumentiert er, dass es solche negativen Folgen für die Religion gar nicht gebe, dass also selbst dann, wenn man die Folgen für die Religion in Betracht zöge, der Materialismus unproblematisch sei.

Aber wie kann der Gedanke von einem Leben nach dem Tode auf materialistischer Grundlage plausibel gemacht werden? Die meisten Materialisten argumentieren ähnlich wie Priestley, indem sie die Auferstehung des Leibes als Garant für ein zukünftiges Leben betrachten. Und diese wird auf unterschiedliche Weise als plausibel angesehen. Priestley beispielsweise verweist auf die Keim-Theorie von Charles Bonnet, die er ebenso wie Hartleys physiologische Psychologie für seine Zwecke fruchtbar zu machen sucht. ${ }^{31}$ Bonnets Theorie besagt, dass jeder Körper aus bestimmten unzerstörbaren »Keimen« bestehe, die für ihn wesentlich seien und die bei allen Veränderungen des Körpers beharren. So sind auch dem menschlichen Leib solche unzerstörbare Keime zuzuschreiben. Diese haben u. a. die Funktion, die Identität des physischen Selbst bei der Auferstehung des Leibes zu garantieren. Durch Bonnets Keim-Theorie wird die Auferstehung als Wirkung von Naturgesetzen erklärbar und ist kein übernatürliches Mysterium mehr. ${ }^{32}$

Auch Hißmann knüpft an Bonnets Keim-Theorie an, um ein Weiterleben nach dem Tode ohne Berufung auf eine Immaterialität der Seele plausibel zu machen.

Es ist ein ganz unbewiesener Satz, dass die materielle Seele zu gleicher Zeit mit verwesen müsse, wenn der grobe Körper zerfällt, weil nicht eine jede Materie nothwendig zerstörbar ist. Bonnets Keime haben sich von Anbegin der Schöpfung in dem Gewühl der Natur herumtreiben lassen, ohne zerstört zu werden, und die Keime, die sich am Ende der Tage entwickeln werden, haben der Zerstückung durch lange Jahrtausende widerstanden. Es giebt sehr feste Körper, die beynahe durch kein Mittel getrennt werden können. ${ }^{33}$

more common, and yet none more blameable, than, in philosophical disputes, to endeavour the refutation of any hypothesis, by a pretence of its dangerous consequences to religion and morality. When any opinion leads to absurdities, it is certainly false; but it is not certain that an opinion is false, because it is of dangerous consequence« (p. 96).

30 Ebd., S. 14.

31 Priestley: Disquisitions (s. Anm. 5), p. 161

32 Bonnet: Essai analytique (s. Anm. 3), \743; Bonnet: La Palingénésie philosophique, on Idées sur l'état passé et sur l'état futur des êtres vivans. 2 Bde. Genf 1769, vol. 1, pp. $311 \mathrm{f}$.

33 Hißmann: Psychologische Versuche (s. Anm. 8), S. 254f. 
Hißmanns Formulierung ist allerdings sehr vorsichtig, denn er verweist lediglich darauf, dass die These von der Vergänglichkeit einer materiellen Seele »unbewiesen« sei. Überdies scheint er sich des spekulativen Charakters von Bonnets Keim-Theorie durchaus bewusst zu sein. Letztlich beruft sich Hißmann denn auch auf die Tradition, nach der die Unsterblichkeit von der Gnade Gottes abhänge und nicht davon, worin das Wesen der Seele bestehe: »Mit eben derselben Kraft, mit welcher Gott eine einfache Monade erhält, kann er eine materielle Substanz ewig leben lassen. Immer kömt es einzig und allein auf seinen gnädigen Willen an, ob er jene in Ewigkeit nicht vernichten und diese durch seine Allmacht ewig unterstützen und beleben werde. $\aleph^{34}$ Hißmann hat mit seiner Argumentation also keine genuin materialistische Lehre von der Unsterblichkeit der Seele begründet, sondern wie schon Locke, Materialität und Unsterblichkeit als miteinander vereinbar gedacht.

Wie erwähnt geht es Hißmann nicht um feine Unterschiede zwischen mehreren Versionen des Materialismus, nicht einmal um einen Beweis für die Materialität der Seele, sondern um die Zurückweisung der traditionellen Lehre von einer immateriellen Seele und darum, die These von der Materialität der Seele als sehr wahrscheinlich nachzuweisen. Was sind nun Hißmanns Argumente, mit denen er versucht, sein Ziel zu erreichen?

\section{Hißmanns Argumentationsstrategien}

Hißmann verfolgt zwei Argumentationsstrategien, ohne diese freilich explizit zu machen. Er versucht, erstens, den Leser von der negativen These zu überzeugen, dass die Seele kein einfaches, immaterielles Wesen sein könne. Zweitens versucht Hißmann, den Materialismus dadurch wahrscheinlich zu machen, dass er Einwände gegen die materialistische Auffassung zu entkräften sucht. Dabei werden einige Argumente von ihm durchaus doppelt für beide Strategien eingesetzt: Er versucht beispielsweise, Einwände gegen den Materialismus zu entkräften, indem er aufzeigt, dass sie auf die immaterialistische Position selbst zutreffen und also diese jedenfalls nicht plausibler machen als die materialistische Auffassung.

\subsection{Einwände gegen die Lehre von der Immaterialität der Seele}

Was diese erste Strategie betrifft, verweist Hißmann (a) auf von ihm behauptete Erfahrungstatsachen, die einen engen Zusammenhang von Seele und Körper nahe legen, und (b) auf immanente Probleme der immaterialistischen Position.

Zu den von Hißmann aufgeführten Erfahrungstatsachen gehören beispielsweise:

i. »Sensibilität und Bewusstsein, Gedächtnis und Einbildungskraft, Verstand und Vernunft« hängen »nach allen Erfahrungen lediglich von gewissen inneren Theilen unseres Körpers«ab.

34 Ebd., S. 255. 
ii. »Die Stärke und Schwäche aller dieser Kräfte« nehmen zu und ab »mit der Stärke des inneren und des äußeren Mechanismus, mit der glücklichen oder unglücklichen Constitution der festen und flüssigen Theile unseres Körpers«.

iii. »Mit der Zerrüttung des Gehirns durch Krankheiten, Alter und Medikamente« ist »der Verlust aller Seelenfähigkeiten unzertrennlich verbunden«. 35

Hißmann meint, mit Hinweis auf solche und ähnliche Zusammenhänge zeigen zu können, dass »keine einzige Erfahrung auf ein einfaches Wesen führt, das vom Gehirn wesentlich verschieden, das das Behältniß aller Empfindungen und Vorstellungen, die der ganze Mensch hat, und der uneingeschränkte Besitzer und Beherrscher aller dieser Reichthümer wäre, die nur Menschen in einer solchen Fülle besitzen. «3 $\mathrm{Da}$ also die Erfahrung eine immaterielle Seele nicht nahe lege, schließt Hißmann:

So kömt mir kein Gedanke, der je von einem Menschen gedacht worden, sonderbarer und unbegreiflicher vor, als der Gedanke von einem einfachen, im Menschen wohnenden Wesen, und die willkührliche Umschaffung des Gehirns in ein einfaches Wesen. ${ }^{37}$

Genauso wenig, wie man auf den Gedanken komme, Gehör, Geruch, Gesicht, Geschmack etc. für Kräfte eines einfachen Wesens zu halten, so wenig hätte man auf den Gedanken verfallen sollen, »Sensibilität, Bewusstsein, Gedächtnis, Einbildungskraft, Verstand, Vernunft zu Kräften eines einfachen Wesens zu machen «. ${ }^{38}$ Dieser Gedanke von der Seele als eines einfachen Wesens sei eine »unwahrscheinliche Fiktion«. Es ist eine Fiktion, die »alle Erfahrungen wider, und keine einzige für sich hat«. ${ }^{39}$

Hißmann berücksichtigt freilich nicht die Tatsache, dass ein Zusammenhang von materiellem Körper und seelischen Kräften von Vertretern der immaterialistischen Auffassung von der Seele (die er abfällig »Simplinisten« nennt) ${ }^{40}$, gar nicht geleugnet wird (Descartes) und auch gar nicht geleugnet werden muss. Hißmann benötigt für seine Argumentation also mehr als den Verweis auf die erwähnten Zusammenhänge und wendet sich vielleicht auch aus diesem Grund (b) den immanenten Problemen der immaterialistischen Position zu.

Hier verweist er (i) auf die bekannten Fragen, die der Substanzdualismus (der eine einfache, immaterielle Seele annimmt) aufwirft. Die »Simplinisten« könnten weder erklären, wie zwei Substanzen ganz heterogener Art (Körper und Seele) interagieren können, noch wie eine immaterielle Seelensubstanz überhaupt individuiert werde.

Wo bleibt z. E. der schöne, einförmige Plan der Schöpfung? Welch ein Sprung von Körper auf Geist, vom Zusammengesezten aufs Einfache? Welche ungefüllte Lücke? Wie reisst die Kette ab? Wie unwahrscheinlich, dass der Mensch aus zween so ganz heterogenen Haupttheilen zusammengesetzt seyn sollte? Wie kann eine Monade an Materie angeklebt seyn, so wie die einfache Seele am äußersten Ende nothwendig mit körperlichen Partikeln zusammenhangen muss? Wodurch kann eine Seele von andern

Hißmann: Psychologische Versuche (s. Anm. 8), S. 248 (alle drei Zitate).

Ebd.

Ebd., S. 248f.

Ebd., S. 249.

Ebd.

Ebd., S. 250. 
unterschieden seyn? Wie kann ein Geist im Körper einen Raum ausfüllen, ohne ausgedehnt zu seyn? Und was heist einfach? - Verneinung, Nichts. ${ }^{41}$

Des Weiteren betont er (ii) nochmals die Tatsache, dass Fälle von »Schwächung und Verlust der Seelenkräfte« durch die Erfahrung mannigfach erwiesen seien und dass diese Tatsache »unmöglich« wäre, wenn die Seelenkräfte »Eigentum eines einfachen Wesens« wären, »das keine Theile einbüßen kann«. ${ }^{42}$

Überdies sei (iii) unerklärbar, wie eine einfache, immaterielle Seele überhaupt empfinden könne. Denn es sei unbegreiflich, »wie ein einfaches Wesen diese Seelenkräfte in sich schliessen, und wie es eine so ungeheure Menge von Ideen verschlingen und wiederum ausspeyen könne«. ${ }^{43}$

Schließlich (iv). stellt Hißmann in Frage, ob wir überhaupt etwas über die Kräfte und die Natur einfacher Wesen erkennen können:

Keiner von unsern äußern oder innern Sinnen macht uns mit ihnen bekannt... Macht man die Seele gar zu einem einfachen geistigen Wesen: so ist bisher weder ein bestimter Begriff von einer geistigen Substanz gegeben, noch aber die Möglichkeit solcher Wesen bewiesen worden. ${ }^{44}$

Dies kann als ein erkenntniskritisches Argument bezeichnet werden. Der Begriff von einem Geist, so Hißmann, ist »ganz negativ«; es ist »weder ein Erfahrungsbegriff, noch eine Vorstellung«, »die leicht nach der Analogie verständlich werden könnte«. ${ }^{45}$ Und selbst wenn wir einen positiven Begriff von einem Geist hätten, sei es noch ein weiter Weg »von der Erklärung der Ingredienzien zum Begriff eines Geistes« zu »dem Satz, dass dergleichen Naturen wirklich oder auch nur möglich seyn «. ${ }^{46}$ Für Hißmann ist dieser Weg noch von niemandem mit Erfolg zu Ende geführt worden.

\subsection{Entkräftung von Einwänden gegen den Materialismus}

Vier Einwände gegen den Materialismus stehen im Zentrum von Hißmanns Diskussion. Dabei sollen die von Hißmann kritisierten Argumente genaugenommen nicht ausschließlich Probleme der materialistischen Position aufzeigen, sondern gleichzeitig positiv für die immaterialistische Auffassung sprechen. Dementsprechend richten sich Hißmanns Gegenargumente auf beide Aspekte dieser Einwände.

Das (i) bereits eingangs erwähnte und von Immaterialisten immer wieder vorgebrachte Argument, dass die Materie wesentlich passiv, »träg und untätig«, sei und ihr daher keine Denk-

41 Ebd., S. 277f. Hißmann verweist hier auf eine Stelle in der Schrift von August W. Hupel: Anmerkungen und Zweifel über die gewöbnlichen Lehrsätze vom Wesen der menscblichen und thierischen Seele. Riga 1774, S. 265.

42 Hißmann: Psychologische Versuche (s. Anm. 8), S. 249.

43 Ebd., S. 259.

44 Ebd., S. 260.

45 Ebd.

46 Ebd., S. 262. 
und andere seelischen Tätigkeiten zugesprochen werden könnten, ${ }^{47}$ versucht Hißmann auf zwei Weisen zu entkräften. ${ }^{48}$

Zunächst behauptet Hißmann, dass es weder bewiesen sei, »dass Denkungsvermögen Thätigkeit sey«, noch, »dass die Materie blos leidend sey« ${ }^{49}$ Wie Priestley verweist Hißmann auf die Tatsache, dass es allgemein üblich ist, der Materie gewisse Aktivitäten und Kräfte zu diesen zuzuschreiben:

Sind Sturmwinde, und Magnetismus, und Elektricismus, und Attraktion, und Gift, und Schiesspulver, und Medikamente bloßes Leiden? Und wenn es bloßes Leiden ist: ist denn alle Materie bloß leidend? Muss nicht eine jede Materie nach ihrer Bestimmung mancherley Kräfte haben? ${ }^{50}$

Hißmann weist also erstens unter Berufung auf Erfahrungstatsachen die These von der wesentlichen Passivität der Materie als unwahrscheinlich zurück. Da wir ihr andere Kräfte zuschreiben, gebe es keinen Grund, der Materie die Denkfähigkeit prinzipiell abzuschreiben. Zweitens verwendet Hißmann wieder ein erkenntniskritisches Argument. Denn mit ihrer These nehmen die „Simplinisten« einfach an, dass das Wesen oder, mit Locke gesprochen, die reale Essenz der Materie erkennbar sei und dass man auch wisse, worin diese reale Essenz bestehe. ${ }^{51}$ Ein solches Wissen sei aber der menschlichen Erkenntnisfähigkeit gar nicht zugänglich. Der immaterialistische Einwand ist also für Hißmann schon aus dem Grund nicht überzeugend, dass er sich

eine Kenntnis der Körperwelt, und der Bestandtheile der Materie anmasst, die Menschenkinder nicht haben können. Wer kennt die Oberfläche genau? Wer kennt die Kräfte des sichtbaren Körperlichen? Und doch sezt man im Beweis voraus, dass wir sogar von den unsichtbaren, einfachen Bestandtheilen der Materie die genaueste Kenntnis besitzen, und von ihren Kräften die die pünktlichste Rechenschaft zu geben im Stande seyn. ${ }^{52}$

Hißmann stellt also keineswegs eine positive Gegenbehauptung über die Materie auf, sondern argumentiert lediglich erstens, dass die Materie, soweit wir sie durch Erfahrung und Beobachtung kennen, Denkfähigkeit jedenfalls nicht ausschließe, und zweitens, dass es unsere prinzipielle Unkenntnis ihres inneren Wesens nicht erlaube, ihr die Denkfähigkeit einfach abzusprechen. Letzteres Argument setzt offensichtlich voraus, dass der Begriff einer denkenden Materie keinen Widerspruch enthalte - eine Position, die an John Locke erinnert, der - wie erwähnt selbst kein Materialist war. ${ }^{53}$

Hißmann macht recht kurzen Prozess mit dem Einwand (ii), wonach der Materialismus die kognitiven Tätigkeiten der Seele nicht erklären könne. Hiernach sei es "ganz unbegreiflich«, »wie ein zusammengesetztes Wesen empfinden, wie es Empfindungen in allgemeine Begriffe verwandeln, wie es sie aufbewahren und wieder hervorrufen, wie es sie in Urtheile und Schlüsse

Ebd., S. 271.

Hißmann erwähnt auf S. 271 seiner Psychologische Versuche (s. Anm. 8) Francis Hutcheson und Johann Georg Sulzer als Vertreter der Auffassung, dass Materie »träg und untätig« sei.

Ebd., S. 271.

Ebd.

Hißmann (ebd., S. 273-277) bezieht sich hierbei kritisch auf Moses Mendelssohns Phädon oder über die Unsterblichkeit der Seele in drey Gesprächen, Berlin und Stettin 1767, (2. Gespräch), und positiv auf Hupels Argumente gegen Mendelssohn in Anmerkungen und Zweifel (s. Anm. 41).

52 Hißmann: Psychologische Versuche (s. Anm. 8), S. $275 f$.

3 Vgl. Locke: Essay (s. Anm. 24), III.vi.4. 
zusammenketten könne«. ${ }^{54}$ Hißmann gesteht zwar zu, dass die Fähigkeit der Bildung allgemeiner Begriffe nicht leicht »aus dem Mechanismus der Werkzeuge unsrer Empfindung erklärbar zu seyn ${ }^{55}$ scheine, aber für unmöglich sei eine solche Erklärung keineswegs zu erachten. Darüber hinaus und grundsätzlicher argumentiert Hißmann erstens, dass selbst, wenn man die Unbegreiflichkeit zugestehe, daraus nicht folge, dass die Seele nicht materiell sein könne. Denn man dürfe »aus der Unbegreiflichkeit gar nicht auf das Nichtdaseyn schliessen ${ }^{56}$ Zweitens falle der Vorwurf auf die immaterialistische Position, und zwar in verschärfter Form, zurück. Denn diese Position könne die Tätigkeiten der Seele ebenso wenig erklären und habe außerdem mit dem bereits erwähnten Problem der Interaktion zweier wesentlich verschiedener Substanzarten zu kämpfen. Daher gilt: »Der Vorwurf der Unbegreiflichkeit trift die entgegengesetzte Hypothese des Materialismus gewiss nicht in einem so hohen Grade. $\aleph^{57}$ Bemerkenswert ist hier die oben erwähnte Betonung des hypothetischen Charakters der materialistischen Position. Bei Materialismus und Immaterialismus handelt es sich für Hißmann um Hypothesen, die nicht nur in Bezug auf die Erfahrungsgrundlage gewürdigt werden müssen, sondern auch vor allem danach zu bewerten sind, ob und wie sie die kognitive Tätigkeiten des menschlichen Geistes erklären können. »Die alte Hypothese des Materialismus« schneidet jedenfalls besser ab als die der »Simplinisten.«

Die letzten zwei wichtigsten Einwände gegen den Materialismus beziehen sich auf die Philosophie des Geistes. Hißmann widmet sich (iii) der oft erhobenen These, der Materialismus sei mit der offensichtlichen Einheit des Bewusstseins nicht vereinbar. Wäre die Seele materiell, so wurde argumentiert, dann hätte die Seele nicht nur kein Bewusstsein ihrer selbst als eines einheitlichen Wesens, sondern könnte auch gar kein einheitliches Wesen sein (wie es aber tatsächlich der Fall sei). Vielmehr müsste man mit dem Materialismus annehmen, dass jedes einzelne materielle Teilchen, aus dem die Seele sich zusammensetzt, für sich besteht und ein eigenes Bewusstsein habe. Denn andernfalls könnte auch aus ihrer Zusammensetzung nicht ein Ganzes entstehen, das des Denkens und des Bewusstseins fähig wäre. Wenn aber jedes Materieteilchen für sich gesondert bewusst wäre, hätten wir es mit einer Vielheit von bewussten Entitäten zu tun, und nicht mit einer Einheit. Es sei aber offensichtlich, dass wir die Seele als ein einheitliches Wesen wahrnehmen und dass sie ein einheitliches Wesen sein müsse, um ihre Tätigkeiten ausüben zu können. Kurz, die Seele könne nicht materiell sein, weil sie dann kein einheitliches Wesen wäre und auch kein einheitliches Bewusstsein produzieren könnte. Diese Kritik der Immaterialisten kann sich darauf berufen, dass auch Materialisten die Einheit der Seele und des Bewusstseins nicht leugnen.

Und in der Tat, Priestley und mit ihm Hißmann sehen die Seele oder den menschlichen Geist als ein einheitliches Wesen an, sie sprechen auch von dem Bewusstsein der Einheit. Allerdings argumentieren sie, und dies sicherlich zu Recht, dass dies ganz und gar nicht unvereinbar ist mit der These, dass die Seele ein materielles Wesen sei. Einheit und Materialität, so argumentieren sie, stellen keinen Widerspruch dar. Die Prämisse der Immaterialisten, dass aus

54 Hißmann: Psychologische Versuche (s. Anm. 8), S. 259.
55 Ebd., S. 265.
56 Ebd., S. 267.
57 Ebd., S. 268. 
unbewussten Materieteilchen kein einheitlich bewusstes Ganzes entstehen könne, sei nicht überzeugend:

Ist denn der Satz so ganz unzweifelhaft, dass aus unbelebten Theilen kein belebtes Ganze, aus undenkenden Theilen kein denkendes Ganze zusammengesezt werden könne? Warum kann aus unharmonischen Theilen kein harmonisches Ganzes werden? Zugegeben, dass einzelne Seelenpartikeln das, was man Vorstellungsvermögen nennt, nicht haben, kann es ihr konzentrirter Zusammenfluss nicht haben $?^{58}$

Es sei vielmehr gerade die Organisation unbewusster materieller Teilchen, die sich zu einem einheitlichen denkenden Wesen fügt. Was für eine Art von Organisation dafür erforderlich sei, entziehe sich zwar unserer Kenntnis, aber es gebe keine guten Gründe, die Möglichkeit einer solchen Organisation nicht anzunehmen. ${ }^{59}$ Jedenfalls sei die Einheit der Seele und das Bewusstsein dieser Einheit keineswegs durch die Materialität der Seele bedroht.

Die Frage nach dem Einheitsbewusstsein führt auf den letzten hier zu betrachtende Einwand, den Hißmann zu entkräften sucht (iv). Hiernach gibt es ein unmittelbares inneres Gefühl, das uns der Einfachheit und damit der Immaterialität der Seele versichere. Träfe dies zu, dann wäre Hißmanns eigener Forderung nach Erfahrungsbegründung entsprochen und seine Behauptung, die Idee von einer immateriellen Seele sei eine solche, die »keine einzige [Erfahrung] für sich hat «, ${ }^{60}$ widerlegt. Hißmann schreibt diese Position Charles Bonnet zu. Denn für Bonnet gelte, dass Denken und Gedanken »zufolge eines innern Gefühls etwas ganz einfaches und untheilbares seyn«, das nur in einem einfachen Subjekt vorkommen könne. ${ }^{61}$

Hißmann argumentiert nun erstens, dass es diese angebliche unmittelbare Erfahrung von Einfachheit gar nicht gebe und dass die Philosophen, die sich für ihre Lehre auf ein solches unmittelbares Einfachheitsgefühl berufen, von ihrem Vorurteil über die Existenz einer einfachen Seele beeinflusst seien. Dies ist mit Hißmanns Hinweis gemeint, dass auch einige empiristisch ausgerichtete Denker, die sich auf innere Gefühle und Beobachtung berufen, von Vorurteilen beeinflusst seien. Sie finden über das Wesen der Seele heraus, was sie finden wollten. »Und so fand man mehrenteils, was man wirklich nicht fand. « ${ }^{62}$ Dass ein jeder das erwähnte Einfachheitsgefühl habe, ist für Hißmann eine bloße Behauptung, die durch die unsere tatsächliche innere Erfahrung nicht gestützt werden könne.

Hißmann scheint zweitens zu argumentieren, dass ein inneres Gefühl, welchen Inhalts auch immer, gar nichts über das Wesen der Seele aussagen könne:

Dass das Gefühl für ein einfaches Wesen zeuge, welches das Substratum des Bewusstseyns sey, ist, da die Sache ein Gefühl betrifft, wo nicht eine gar falsche, doch eine sehr zweifelhafte Voraussetzung. ${ }^{63}$

Ebd., S. 276.

Ebd., S. 270. Priestley's Argumentation ist expliziter formuliert als Hißmanns: „Surely there may be a separate unity of the whole nervous system, as well as of one atom; and if the perception that we call consciousness, or that of any other complex idea, necessarily consists in, or depends upon, a very complex vibration, it cannot possibly belong to a single atom, but must belong to a vibrating system, of some extent (Priestley: Disquisitions [s. Anm. 5], p. 87).

Hißmann: Psychologische Versuche (s. Anm. 8), S. 249.

Ebd., S. 272.

Ebd., S. 247.

Ebd., S. $272 f$. 
Dieser Gedanke entspricht Priestleys Argumentation, die ausführlicher als Hißmanns ist, auf die sich dieser hier aber nicht explizit bezieht. Das Wesen des denkenden Subjekts, argumentiert Priestley, könne nicht durch das innere Bewusstsein und ein inneres Gefühl erkannt werden. Das Bewusstsein sei ein »internal feeling«, durch welches wir von dem wissen, "what passes within our own minds«, also Gedanken und Gefühle. ${ }^{64}$ Unsere Überzeugungen von dem Wesen des Subjekts dieser Gedanken und Gefühle seien aus dem, was unmittelbar im Bewusstsein gegeben ist, erschlossen, und nicht durch innere Gefühle selbst zugänglich. ${ }^{65}$

Drittens argumentiert Hißmann in Erweiterung des zweiten Arguments, dass selbst dann, wenn es ein Gefübl der Einfachheit gäbe, daraus nicht folge, dass das ihm zugrunde liegende Subjekt in einem metaphysischen Sinne ein einfaches Wesen sein müsse. Die Einfachheit des Bewusstseins, wenn wir diese einmal annehmen, könne durchaus aus einem zusammengesetzten, also materiellen Wesen hervorgehen.

Wenn aber auch das Bewustseyn wirklich etwas untheilbares wäre: so muss das Principium des Bewusstseyns deswegen nicht auch ein untheilbares Wesen seyn. Dieses kann zusammengesezt seyn, und demungeachtet ungetheilte, einfache Empfindungen und Gedanken haben, wenn anders diese unverständlichen Worte etwas bedeuten sollen. ${ }^{66}$

Es sei durchaus möglich, argumentiert Hißmann, dass »viele Gehirnfibern [...] zur Erzeugung eines einfachen Gedankens zusammenwirken «. ${ }^{67}$ Jedenfalls spreche nichts gegen diese Möglichkeit. Auch hier stimmen Hißmann und Priestley überein. Allerdings konzediert Priestley anders als Hißmann, dass es über das Bewusstsein von Gedanken und Handlungen hinaus auch ein Gefühl der Einheit gebe, doch dieses könne, so sagt Priestley dann genau wie Hißmann, aus einer zusammengesetzten Entität hervorgehen. Er unterscheidet also die These von der Einheit des Bewusstseins von der Einfachheitsthese in Bezug auf das denkende Subjekt. Aus jener Einheit sei nicht auf die Einfachheit des Ich oder die Immaterialität der Seele zu schließen. Das Einheitsgefühl erlaube es durchaus, dass das Subjekt ein materielles Wesen sein könne. Was aus diesem Einheitsgefühl geschlossen werden könne, sei nur dies, dass man nur eine Person, ein empfindendes und denkendes Wesen sei und nicht zwei Personen oder zwei empfindende Wesen. ${ }^{68}$ Zwar könne in der Tat ein empfindendes Wesen nicht in zwei empfindende Wesen aufgeteilt werden, da eine solche Teilung das eine »system of intelligence which we call the soul of man

64 Joseph Priestley: Illustrations of Some Particulars in the Disquisitions on Matter and Spirit. In: A Free Discussion of the Doctrines of Materialism, and Philosophical Necessity, in a Correspondence between Dr. Price and Dr. Priestley. London 1778, pp. 229-320, hier p. 280.

65 "What we feel, and what we do we may be said to know by intuition; but what we are we know only by deduction, or inference from intuitive observations« (Priestley: Illustrations [s. Anm. 64], p. 283). Schon Hartley hatte das Argument in Frage gestellt, nach dem die Immaterialiät der Seele sich aus der Einheit des Bewusstseins ergebe. Aber Hartley stellte anders als Priestley sogar in Frage, ob der Ausdruck »Einheit des Bewusstseins« überhaupt einen bestimmbaren Sinn ergebe. Hartley: Observations on Man (s. Anm. 3), p. 321: »It is difficult to know what is meant by the unity of consciousness. « Zu Hartleys allerdings sehr knapper Diskussion dieses Themas vgl. Richard C. Allen: David Hartley on Human Nature. Albany, NY 1999, pp. 380-81.

66 Hißmann: Psychologische Versuche (s. Anm. 8), S. 273.

67 Ebd., S. 273.

68 Priestley sagt, es gebe »a feeling or perception of the unity of my nature or being; but all that can be inferred from this is, that I am only one person, one sentient and thinking being, and not two persons, or two sentient or thinking beings« (Priestley: Disquisitions [s. Anm. 5], p. 86). 
zerstören würde, aber natürlich könne die Seele des Menschen so geteilt oder aufgelöst werden »as to become no system of intelligence at all ${ }^{69}$

Hißmann lehnt, wie erwähnt, im Gegensatz zu Priestley den Gedanken eines Gefühls der synchronischen Einheit des Subjekts ab, nicht zuletzt, weil er befürchtet (aber unbegründeter Weise), damit den Immaterialisten ein zu großes Zugeständnis zu machen. ${ }^{70}$ In einem anderen Sinne spricht aber auch Hißmann von einem Einheitsgefühl, nämlich von dem Gefühl der diachronen Einheit oder der Einheit der Person. »Das Gefühl der Einheit seiner Person« kann niemandem »abgesprochen werden ${ }^{71}{ }^{71}$ Dies bedeutet aber nur, dass ich mich durch meine Erinnerungsfähigkeit auf vergangene Handlungen und Gedanken beziehe und diese mir jetzt selbst zuschreiben kann. Für dieses Einheitsgefühl ist also eine Synthesis von gegenwärtiger und vergangener Erfahrung erfordert. Damit ist aber nichts zugestanden in Bezug auf das metaphysische Wesen des Ich. Hißmann weist allerdings die Behauptung zurück, dass wir nicht nur ein Gefühl der diachronen Einheit hätten, sondern auch ein solches der numerischen Identität über die Zeit hinweg. Dieses sei mit der Veränderbarkeit des Ich nicht vereinbar und sogar "physisch unmöglich «. ${ }^{72}$ Auch hier dürfte als Motiv im Hintergrund stehen, dass man mit dem Zugeständnis eines Gefühls numerischer Identität (die keine Veränderung gestatte) dem Immaterialismus ein (nicht erforderliches) Zugeständnis machen würde.

\section{Materialistische Metaphysik und Subjektivität}

Wir haben bereits betont, dass es Hißmann trotz seiner teilweise radikal anmutenden Rhetorik nicht um einen Beweis der Materialität der Seele geht, sondern nur darum, diese wahrscheinlich zu machen. Aber auch bei diesem recht bescheidenen Ziel bleiben noch einige Fragen offen. Auf einige Probleme ist schon bei der Analyse von Hißmanns Vorgehen und Argumentation hingewiesen worden. Dazu gehört beispielsweise die Tatsache, dass Hißmann vage bleibt, wenn es darum geht, das Verhältnis von physischer Basis und mentalen Zuständen zu bestimmen.

Dies ist verknüpft mit der Frage nach dem Subjektbegriff. Wie wird von Hißmann das Subjekt des Denkens und Empfindens bestimmt? Auch hier scheint seine Position nicht eindeutig bestimmbar zu sein. Einerseits betont er bisweilen streng materialistisch, dass »unsere Nerven, eine sichtbare körperliche Substanz, empfinden «, ${ }^{73}$ dass also die Nerven selbst Subjekt der Empfindung seien. Aber Hißmann ergänzt sogleich: »[U]nd wenn sie mit einem andern körperlichen Teil des Menschen zusammenhangen: so sind wir uns der Empfindung bewust «. ${ }^{74}$ Wer ist hier das Subjekt dieses Bewusstseins? Die »Nerven« scheint Hißmann selbst mit seiner Formulierung als Kandidaten auszuschließen. An anderen Stellen suggeriert Hißmann, dass der "ganze Mensch« als das Subjekt des Denkens und Empfindens anzusehen sei, beispielsweise

69 Priestley: Disquisitions (s. Anm. 5), p. 87.

70 Hißmann: Psychologische Versuche (s. Anm. 8), S. 156f. Vgl. hierzu Udo Thiel: Varieties of Inner Sense. Two Pre-Kantian Theories. In: Archiv für Geschichte der Philosophie 79 (1997), pp. 58-79, hier pp. $74 \mathrm{f}$.

71 Hißmann: Psychologische Versuche (s. Anm. 8), S. 151.

72 Ebd. S. 148. Vgl. Thiel: Varieties of Inner Sense (s. Anm. 70), S. 76.

73 Hißmann: Psychologische Versuche (s. Anm. 8), S. 263.

74 Ebd. 
wenn er von »Empfindungen und Vorstellungen« spricht, »die der ganze Mensch hat «, ${ }^{75}$ aber dies wird nicht weiter erläutert oder ausgeführt. Auf den Personbegriff ist schon hingewiesen worden. Wir sahen, dass es dabei um die diachrone Einheit (nicht numerische Identität) des Subjekts geht. Auch diese versucht Hißmann bisweilen materialistisch zu deuten:

Person oder Ich sind weiter nichts, als diejenigen Organen in uns, die neue Eindrücke, Vorstellungen und Handlungen mit den ehemaligen, die diesen neuen ihre Auferstehung zu verdanken haben, vergleichen, und sich dadurch ihrer vormaligen Existenz bewußt sind. Daher sind wir auch nicht dieselben Personen, die wir vorher waren. ${ }^{76}$

Hier wird das Ich oder die Person mit den »Organen« identifiziert, die Vergleichungshandlungen vornehmen und sich dadurch ihrer vergangenen Existenz bewusst werden, also Subjekt des Bewusstseins sein sollen. Andererseits wird die Personalität oder die personale Einheit von Hißmann auch ganz unabhängig von der materiellen Basis mentaler Akte beschrieben: Wir haben das Gefühl der Personalität, sagt Hißmann, »wenn wir nicht blos fühlen, daß wir jezt sind, sondern auch, daß wir ehedem in den vorigen Zeitpunkten unsers Lebens existirten «. ${ }^{77}$ Es wird deutlich, dass die materialistische Metaphysik zum Verständnis dessen, was Person bzw. personale Einheit ist, gar nicht erforderlich ist und auch gar nichts dazu beiträgt. Es ist uns unmittelbar durch ein Bewusstsein oder ein Gefühl gewahr:

Wir fühlen die Einheit [...] unsrer Person immer, wenn wir während eines gewissen Zeitraums unsers Lebens uns gewisser Empfindungen, Vorstellungen und Handlungen bewust sind, und dabey uns bewust sind, daß wir die Eindrücke empfunden, die Vorstellungen gehabt, und die Handlungen ausgeübt haben. $^{78}$

Diese Hinweise verweisen auf ein Problem, das Hißmanns Projekt im Ganzen betrifft. Hißmann sagt zu Beginn seiner Psychologischen Versuche, man könne die ersten vier Versuche akzeptieren, ohne den materialistischen Schluss im 5. Versuch zu ziehen. ${ }^{79}$ Und in der Tat handelt es sich etwa im dritten Versuch über den inneren Sinn bei aller rhetorischen Berufung auf materielle Grundlagen hauptsächlich um begriffliche Analysen (was ist »Selbstbewusstsein«? Was ist »Personalität?« usw.), für deren Behandlung die Frage danach, ob die Seele materiell ist oder nicht, offen gelassen werden kann. Hißmann weist in diesem Zusammenhang auch darauf hin, dass die physische Basis des inneren Sinns uns ohnehin unbekannt sei:

Uns ist eine genaue Kenntnis der Organen des inneren Sinnes gänzlich versagt [...]. Wir kennen die Art gar nicht, wie auf diese inneren Organen von innen Impressionen geschehen, deren Erschütterungen wir unsere inneren Gefühle und Empfindungen zu danken haben. ${ }^{80}$

Also, so könnte man hinzufügen, kann man sich auf diese unbekannten »Organen« auch nicht als Erklärungsgrundlage beziehen. Hißmann unterminiert letztlich seine eigene zentrale These, man müsse als Philosoph (oder »Psycholog«) notwendig »Physiolog und Anatom« sein, ${ }^{81}$ da die

\footnotetext{
75 Ebd., S. 248.

76 Ebd., S. $148 f$.

77 Ebd., S. 145.

78 Ebd., S. 151.

79 Ebd., S. 12 f.

80 Ebd., S. 104f.

81 Ebd., S. 11.
} 
Untersuchung sich auf den Geist oder die Seele als materielles Wesen beziehen müsse. Die Hauptteile seiner Versuche sind gar nicht an eine materialistische Lehre vom Wesen der Seele gebunden. Kurz, wesentliche Aspekte von Hißmanns eigener Philosophie des Geistes unterlaufen seinen offiziell deklarierten Materialismus. 
Bereitgestellt von | De Gruyter / TCS 